\title{
ANÁLISIS ESTÁTICO NO LINEAL DE EDIFICIOS APORTICADOS DE HORMIGÓN ARMADO APLICANDO NORMATIVA ECUATORIANA, COLOMBIANAY PERUANA
}

\section{NON-LINEAR STATIC ANALYSIS OF RC FRAME BUILDINGS USING ECUADORIAN, COLOMBIAN AND PERUVIAN CODES.}

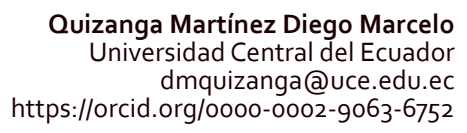

Quizanga Martínez Diego Marcelo Universidad Central del Ecuador dmquizanga@uce.edu.ec https://orcid.org/0000-0002-9063-6752

\author{
Viera Arroba Luisa Paulina \\ Universidad Central del Ecuador \\ Iviera@uce.edu.ec \\ https://orcid.org/oooo-0003-0434-7979
}

\author{
Andino Carranco José Luis \\ Universidad Central del Ecuador \\ jlandino@uce.edu.ec \\ https://orcid.org/0000-0001-6612-6333
}

\section{RESUMEN}

Ecuador está localizado en una zona de alto peligro sísmico, si consideramos adicionalmente que muchas de las estructuras en el país son vulnerables se infiere que el riesgo sísmico en Ecuador es elevado. Para reducir este riesgo se puede modificar la vulnerabilidad de las estructuras, en este sentido los códigos de construcción establecen los requerimientos mínimos para obtener estructuras que sean seguras para sus usuarios. Sin embargo, no hay muchos estudios que comparen la vulnerabilidad de estructuras diseñadas con la norma ecuatoriana de la construcción (NEC-15) respecto a la vulnerabilidad que se obtendría usando otros códigos. Entender el grado de vulnerabilidad de estructuras de hormigón armado diseñadas con NEC-15 respecto a la vulnerabilidad obtenida usando la norma de países vecinos nos permitirá sugerir recomendaciones a nuestra actual norma. Para ello realizamos 18 modelos de edificios de hormigón armado, considerando los diferentes parámetros indicados en las normas (NEC2015), el Reglamento Colombiano de Construcción (NSR-10) y el Reglamento Nacional de Edificaciones de Perú (E.030) y a partir de este diseño realizamos un análisis estático no lineal para posteriormente mediante el método del espectro de capacidad evaluar la vulnerabilidad de estas estructuras. Del conjunto de estructuras estudiadas determinamos que la aplicación de la NSR-10 reflejo un grado de vulnerabilidad menor pese a que los edificios diseñados con E.030 fueron más rígidos, además determinamos que las estructuras diseñadas en las mismas condiciones con NEC-15 son más vulnerables que las diseñadas usando NSR-10 y E030, pese a ello, las respuestas encontradas pueden variar incluyendo si se incluyen otras configuraciones estructurales lo cual se sugiere como un trabajo futuro.

Palabras clave: diseño estructural; vulnerabilidad estructural; análisis estático no lineal; riesgo sísmico.

\section{ABSTRACT}

Ecuador is located in a high seismicity zone. If we consider that many Ecuadorian structures are vulnerable, it is evident that the seismic risk in Ecuador is high. If the structure's vulnerability is reduced the seismic risk will decrease, in this context the Building Construction Codes regulate the minimum requirements to obtain safe structures for their users, however, there are not many studies comparing the vulnerability obtained using Ecuadorian Construction Code (NEC15) regarding the vulnerability obtained using other Constructions Codes. To understand the degree of vulnerability of reinforced concrete structures designed with NEC-15 has regarding 
those designed using National Construction Codes of Ecuador's nearby countries, will allow us to recommend improvements to NEC-15. To do that, we designed 18 reinforced concrete building using NEC-15 and Colombia and Peru Construction Codes (NSR10 and E030, respectively). Considering the recommendations of these 3 National Constructions Codes, we obtain parameters required to carry out a nonlinear static analysis and from this data, we assessed the vulnerability of these buildings using the capacity spectrum method. We concluded that the application of the NSR-10 reflects a lower degree of vulnerability in the structures, even though buildings designed using E.030 were more rigid, also we found that structures designed under the same conditions with NEC-15 are more vulnerable than structures designed using NSR and E030. Still that, this vulnerability may change if other structural configurations are used, which we suggested as future work.

Keywords: structural design; structural vulnerability; non-linear static analysis; seismic risk.

\section{INTRODUCCIÓN}

A lo largo de la historia del Ecuador, grandes movimientos de suelo han ocurrido en el país, siendo hasta ahora el sismo de Esmeraldas de 1906 el evento de mayor magnitud ocurrido, éste es uno de los 10 eventos de mayor magnitud ocurridos en la historia de la humanidad. Según Aguiar (2008), el Ecuador se encuentra en la zona de mayor peligrosidad sísmica del mundo, de tal forma que los profesionales encargados del diseño estructural de edificaciones deben considerar la acción sísmica para la planificación de estructuras. Se debe tener en cuenta que un sismo no mata a las personas sino las edificaciones que colapsan ante un evento de gran magnitud, generalmente estas edificaciones no han sido diseñadas ni construidas respetando los procesos y normativas vigentes.

Sabiendo que el riesgo sísmico depende de la vulnerabilidad de la estructuras y del nivel de amenaza sísmica, este investigación se enfocó en estudiar la vulnerabilidad la misma que está ligada íntimamente con las recomendaciones exigidas por la norma de construcción NEC-2015, se realizó un análisis estático no lineal de una edificación tipo, de cinco pisos de hormigón armado, utilizando la norma ecuatoriana de la construcción (NEC-2015), el reglamento colombiano de la construcción (NSR-10) el reglamento nacional de edificaciones (E-030 PERU), se tuvo como resultado 18 modelos estructurales de edificios, en los que se considera la zonificación sísmica, el tipo de suelo y los factores de seguridad que considera cada norma, con el objeto de determinar la incidencia de aplicación de una u otra normativa en el desempeño de las edificaciones analizadas.

La importancia de este estudio radica en que, a partir de las conclusiones obtenidas, los entes encargados de regular la construcción en los países analizados pueden discutir acerca de las diferencias en los niveles de vulnerabilidad que produce la aplicación de una u otra norma en una misma edificación asentada sobre suelo de condiciones similares y en una zona geográfica próxima y a partir de esto establecer parámetros que permitan tener estructuras más seguras.

La noche del 16 de abril se produjo un sismo de magnitud 7.8 Mw, en el Cantón Pedernales, en la Provincia de Manabí, se pudo apreciar que varias edificaciones fueron reducidas a escombros, mostrando múltiples deficiencias en los sistemas constructivos, por ello la rotunda necesidad de seguir investigando y mejorar las normativas nacionales.

Actualmente en el Ecuador existen investigaciones como la de Arciniegas \& Suárez (2016) con su tesis "Análisis Comparativo Económico - Estructural de Edificios de 6, 12 y 18 pisos, aplicando el Código Ecuatoriano de la Construcción (CEC-2000) y la Norma Ecuatoriana de la Construcción (NEC-15)", y la de Jaramillo \& Rocha (2013) con su tesis 
"Comparación entre la Norma Ecuatoriana de la Construcción (NEC-11) y la Norma American Society of Civil Engineers (ASCE07-10) para diseño Sismo - Resistente: Corte Basal". Estos trabajos principalmente analizan aspectos de diseño y costos, tomando en cuenta los peligros sísmicos a los que el país se encuentra expuesto por su ubicación geográfica. Concluyeron dichos estudios que con la aplicación de la NEC-15 se obtienen secciones menos esbeltas que usando el CEC2000. También se determinó que, dentro del aspecto económico, la NEC-15 representa un mayor costo constructivo, y que el nivel de exigencia en los procedimientos sismo resistentes es mayor en la ASCE 7-10 con relación a la NEC-15.

Sin embargo, no existe ninguna investigación que contemple una comparación del desempeño obtenido de una misma estructura luego de aplicar los códigos de construcción de tres países con similares condiciones de peligrosidad sísmica como son Colombia, Perú y Ecuador. En el presente trabajo se ha realizado análisis estáticos lineales y no lineales, para obtener curvas de capacidad y puntos de desempeño que permitan determinar la vulnerabilidad de 18 modelos, a partir de una estructura aporticada de 5 pisos con la misma configuración geométrica, para dicha estructura se obtuvieron diferentes dimensiones de los elementos estructurales en virtud de los requerimientos de cada código, se analizaron y diseñaron estructuras para diferentes tipologías de suelo y de acuerdo a la zonificación sísmica mostrada en cada código. Para que la ubicación geográfica y el peligro asociado a esta ubicación no incida en los resultados, el análisis de las estructuras se lo realizó considerando que estarían localizadas en provincias fronterizas entre los países, por lo que se modeló estructuras localizadas en San Lorenzo y Tumaco para comparar el desempeño de estructuras diseñadas con NEC-15 y NSR10, así también se consideraron estructuras ubicadas en Huaquillas y Aguas Verdes, para comparar el desempeño de estructuras diseñadas con las normas NEC-15 y E-30. La ubi- cación de los modelos realizados asi como los mapas de zonificación sísmica de los tres países que se consideraron en este estudio, se pueden apreciar en la figura 1 hay una distinción por colores en base al peligro sísmico que muestran las normas de los 3 países.

Adicionalmente se debe indicar que la edificación que se consideró para el diseño es de una tipología estructural que atiende a la generalidad de construcciones que se realizan actualmente en el Ecuador: estructura aporticada de cinco pisos y de hormigón armado (material más usado en la construcción en Ecuador, Colombia y Perú).

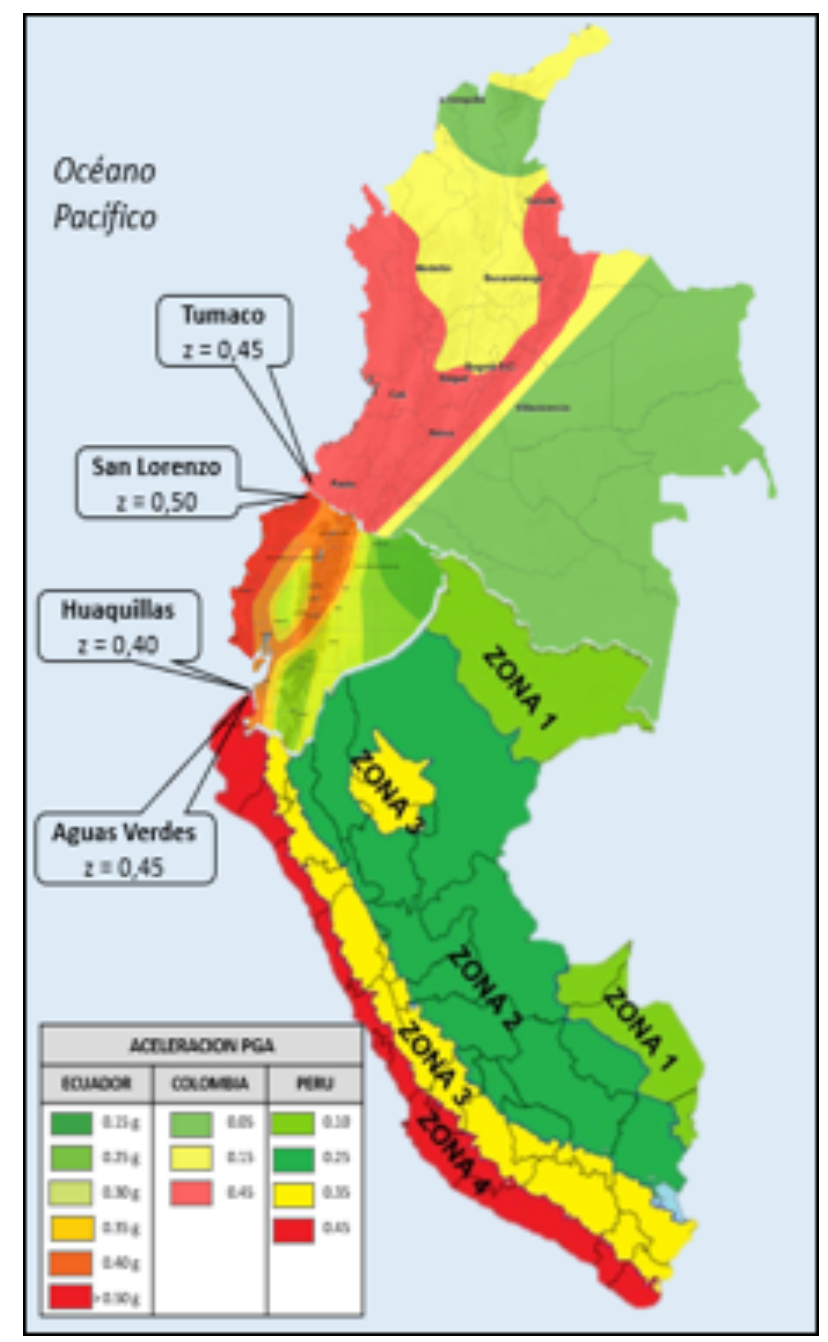

Figura 1 Mapa de zonificación sísmica de Colombia, Ecuador y Perú. Fuente: NEC-15, NSR-10 Y E.030

De acuerdo con lo que indica Llano (2015) y Santiago Vásconez, Técnico del "Departamento de Revisión y Aprobación de Planos Estructurales de la Entidad Colaboradora de 
Pichincha", más del 95\% de cálculos estructurales, correspondientes a edificios, que se realizan en la ciudad de Quito, se basan en un análisis lineal controlado por derivas inelásticas, es decir no se realiza ningún otro tipo de verificación o comprobación del cálculo estructural. Sin embargo, los desastres que han ocurrido por causas sísmicas a lo largo de la historia del país demuestran que un análisis lineal controlado por derivas inelásticas no es suficiente para asegurar que las estructuras tengan un buen comportamiento sísmico, es necesario avanzar a otras técnicas que permitan prever el desempeño de una estructura, y lograr controlar los niveles de daño estructurales y no estructurales.

\section{METODOLOGÍA}

En esta investigación se consideró una edificación de cinco pisos y cuatro vanos separados a 5 metros en los dos sentidos (ver Figura 2) por ser una estructura regular en planta y elevación, la estructura fue sometida a un análisis estático lineal y posteriormente diseñada cumpliendo con las recomendaciones sismo resistentes dispuestas por las normas vigentes en Ecuador, Colombia y Perú.

A partir de las estructuras diseñadas se realizó un análisis estático no lineal (Nolinear-Static-Pushover), mediante el método de zonas plásticas.

Se comparó el desempeño de estructuras luego de ser diseñadas usando las normativas de 3 países vecinos (Ecuador, Colombia y Perú), a través de analizar una configuración geométrica igual en todos los casos. De esta manera se definieron 18 modelos que están en función del tipo de suelo: Ecuador (suelos A, B, C, D, E) vs Colombia (suelos A, $B, C, D, E$ ) y Ecuador (suelos $A, B, D, E$ ) vs Perú (suelos S0, S1, S2, S3).
La localización de las estructuras se supuso en puntos fronterizos de cada país, señalados previamente, donde se infiere características geológicas y sísmicas similares, de acuerdo con las normas vigentes aplicadas en cada país. La descripción de los 18 modelos se puede apreciar en la Tabla 1.

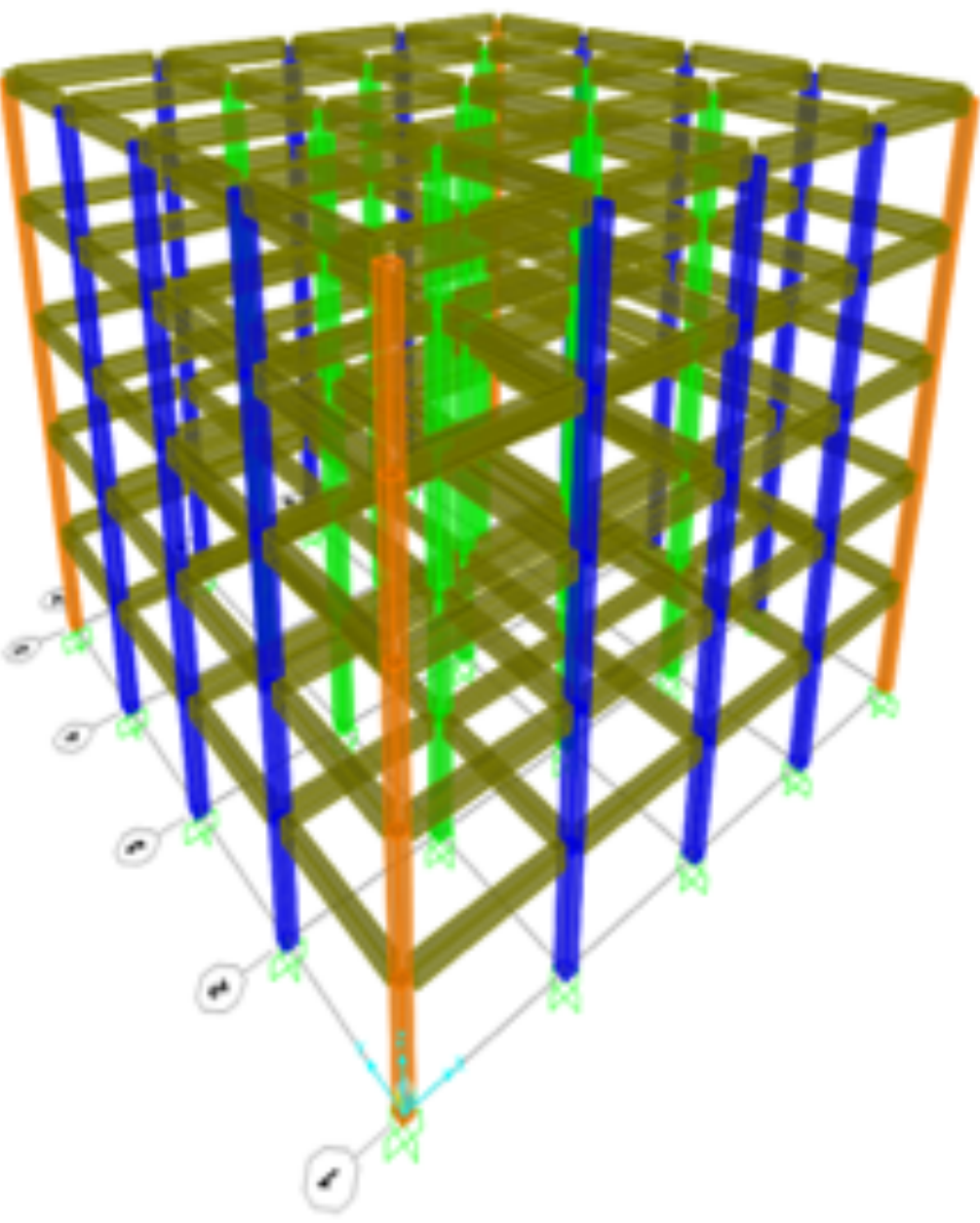

\section{Figura 2 Estructura tipo / Fuente: SAP2000}

Los modelos descritos en la Tabla 1 se analizaron, por medio de un programa computacional de análisis y diseño estructural (SAP2000), considerando las diferencias entre los parámetros de diseño de las normativas aplicadas, y posteriormente ya realizado el modelado se determinaron: periodos de vibración, derivas de piso, curvas de capacidad y puntos de desempeño de cada modelo. 
Tabla 1. Nomenclatura de los modelos

\begin{tabular}{|c|c|c|c|c|}
\hline PAIS & MODELO & $\begin{array}{l}\text { TIPO DE } \\
\text { SUELO }\end{array}$ & UBICACIÖN & $\begin{array}{l}\text { NORMATIVA DE } \\
\text { DISENNO }\end{array}$ \\
\hline \multirow{9}{*}{ ECUADOR } & Model0 1 & A & San Lorenzo & NEC-15 \\
\hline & Modelo 2 & B & San Lorenzo & NEC-15 \\
\hline & Modelo 3 & C & San Lorenzo & NEC-15 \\
\hline & Modelo 4 & D & San Lorenzo & NEC-15 \\
\hline & Modelo 5 & $E$ & San Lorenzo & NEC-15 \\
\hline & Modelo 6 & A & Huaquillas & NEC-15 \\
\hline & Modelo 7 & B & Huaquillas & NEC-15 \\
\hline & Modelo 8 & D & Huaquillas & NEC-15 \\
\hline & Modelo 9 & $E$ & Huaquillas & NEC-15 \\
\hline \multirow{5}{*}{ COLOMBIA } & Modelo 10 & A & Tumaco & NSR-10 \\
\hline & Modelo 11 & B & Tumaco & NSR-10 \\
\hline & Modelo 12 & C & Tumaco & NSR-10 \\
\hline & Modelo 13 & D & Tumaco & NSR-10 \\
\hline & Modelo 14 & $E$ & Tumaco & NSR-10 \\
\hline \multirow{4}{*}{ PERU் } & Modelo 15 & So & Aguas Verdes & E. 030 \\
\hline & Modelo 16 & S1 & Aguas Verdes & E. 030 \\
\hline & Modelo 17 & S2 & Aguas Verdes & E. 030 \\
\hline & Modelo 18 & S3 & Aguas Verdes & E.030 \\
\hline
\end{tabular}

\section{DISCUSIÓN DE RESULTADOS}

La NEC-15 establece que la deriva máxima para cualquier piso no excederá los límites dispuestos (2\%), mientras la NSR-10 dispone un parámetro del $1 \%$ para estructuras de concreto armado, sin embargo, este estudio consideró que para la comparación del desempeño las estructuras deberían tener desplazamiento de piso relativos iguales al límite máximo permitido por cada norma más una tolerancia adicional de $0.1 \%$.

El Reglamento Nacional de Edificaciones E.030 Perú, es el más riguroso referente a la deriva permitida, ya que establece un valor de deriva permisible de hasta el $0.7 \%$. Resulta evidente que debido a derivas bajas las estructuras modeladas usando la normativa peruana resultaran más rígidas.

Una vez establecidas las secciones de los modelos y luego de verificar su resistencia de acuerdo con cada normativa, se obtuvo de cada modelo las derivas de piso las cuales según se pueden apreciar en las figuras 3 y 4 están dentro de los límites máximos considerados para para este estudio.



Figura 3. Derivas de piso NEC-15 vs. NRS-10

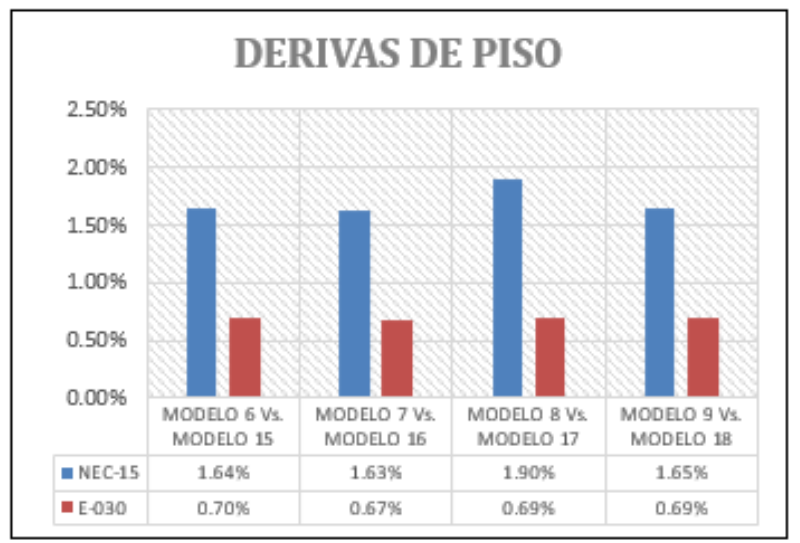

Figura 4. Derivas de piso NEC-15 vs. E.030

Luego de realizar el análisis estático lineal y una vez obtenidas las secciones de hormigón armado de cada elemento estructural se realizó un análisis estático no lineal de todos los modelos tridimensionales usando los coeficientes de FEMA 356, se logró estimar la curva de capacidad de los 18 modelos y se presentan a continuación. Estas curvas muestran la relación entre el desplazamiento en el techo de los edificios y la fuerza Basal.

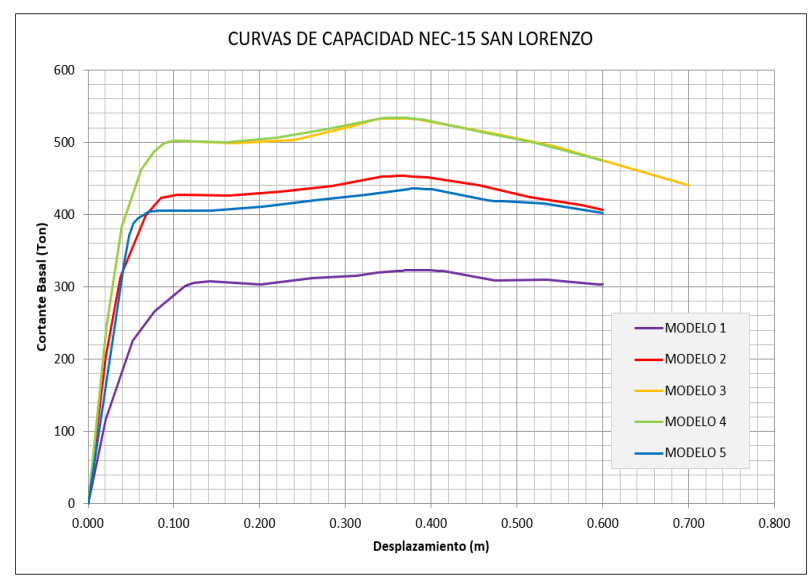

Figura 5. Curvas de capacidad San Lorenzo 


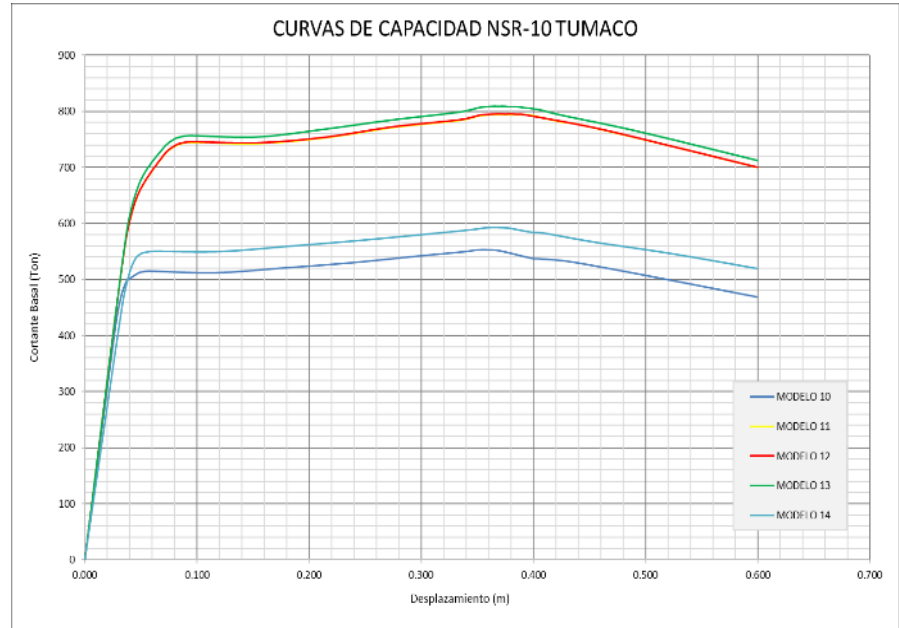

Figura 6. Curvas de capacidad Tumaco

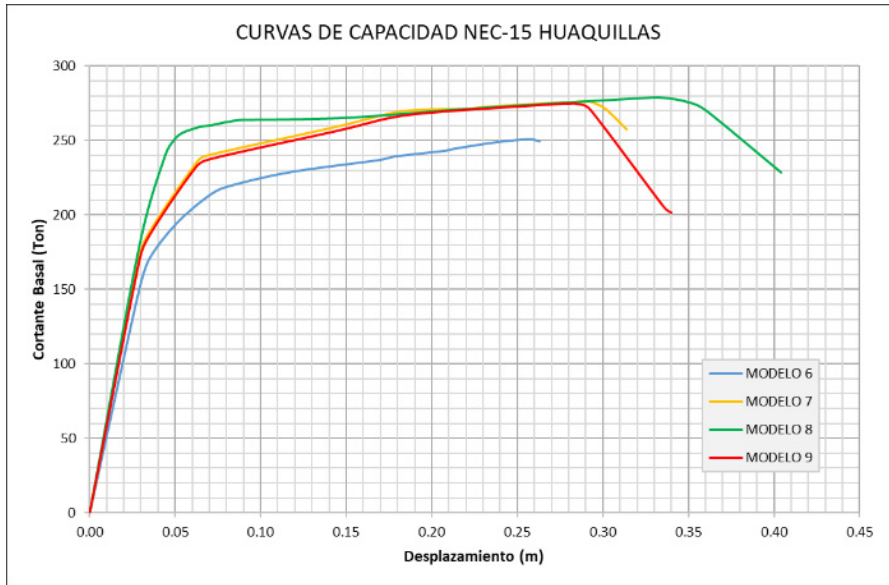

Figura 7. Curvas de capacidad Huaquillas

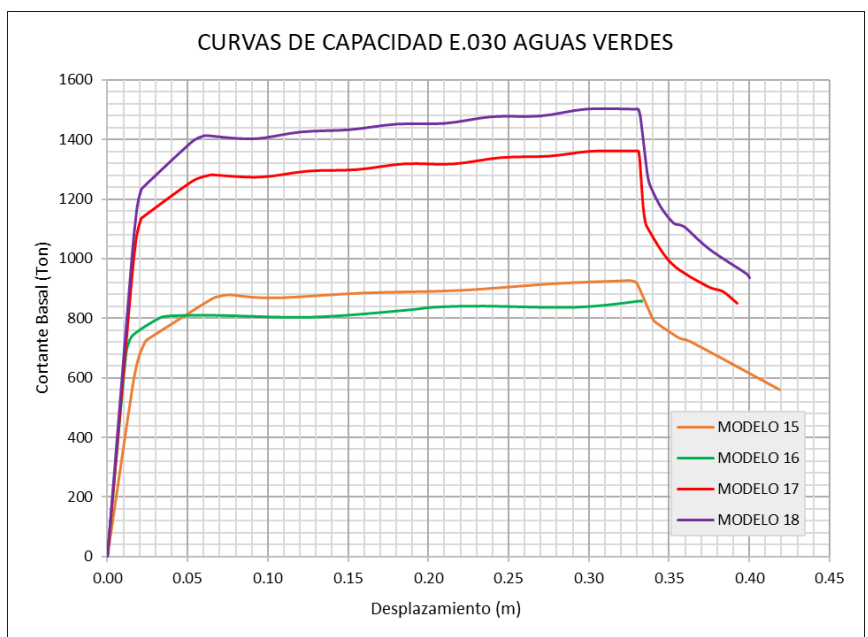

Figura 8. Curvas de capacidad Aguas Verdes

Dentro del análisis de las Curvas de Capacidad se tiene en consideración que los modelos desarrollados con la NEC-15 (ver figuras 5 y 7), presentan una menor capacidad de resistencia estructural, puesto que los valores de capacidad de los modelos desarrollados con la NSR-10 y E.030 son mayores (ver figuras 6 y 8), quedando claro en este estudio que la normativa ecuatoriana es la más permisiva, esto es un resultado lógico teniendo en cuenta el valor del porcentaje admisible de derivas de piso $(2 \%)$, en comparación con Colombia (1\%) y Perú $(0.7 \%)$.

Hay que tener en consideración que el nivel de desempeño puede obtenerse a partir de la sectorización de la curva de capacidad se tiene así los siguientes límites "Ocupación Inmediata" (IO) y "Seguridad de Vida" (LS) y Prevención de Colapso (CP) (ver figura 9). Referente a estructuras no esenciales las normas analizadas no exigen tener niveles de desempeño de ocupación inmediata, pero si buscan garantizar al menos el nivel de Seguridad de Vida.

La figura 9 muestra esquemáticamente la curva de capacidad, reflejando los umbrales de desplazamiento lateral y estados límite considerados por los lineamientos FEMA.

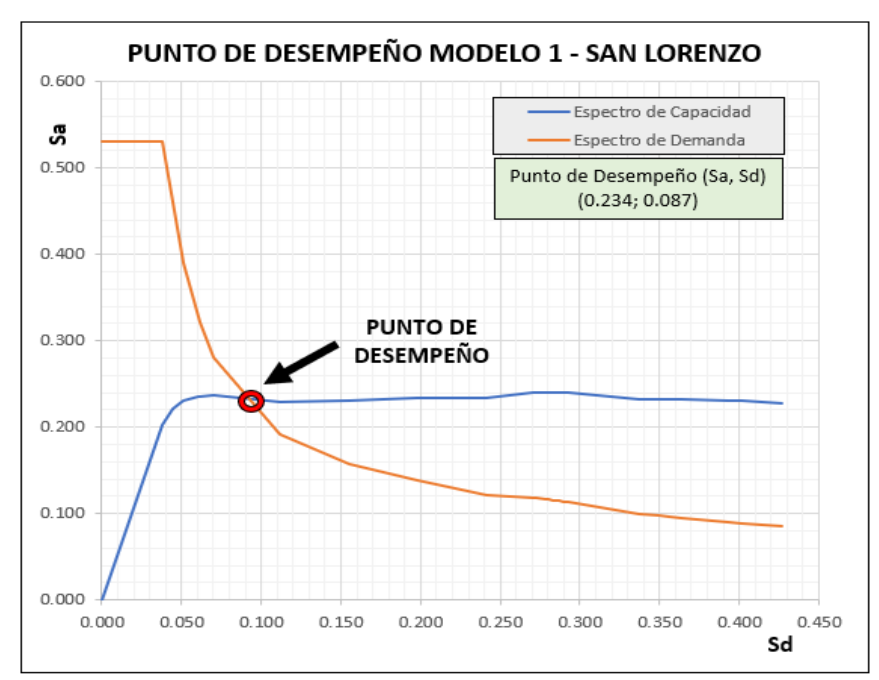

Figura 9 Curva de capacidad y estados límit

Una vez definidas las curvas de capacidad y de demanda, se obtuvieron los puntos de desempeño de los 18 modelos, los mismo que representan el máximo desplazamiento probable para una determinada demanda sísmica, se aprecia por ejemplo en el figura 10 el punto de desempeño del modelo 1. 


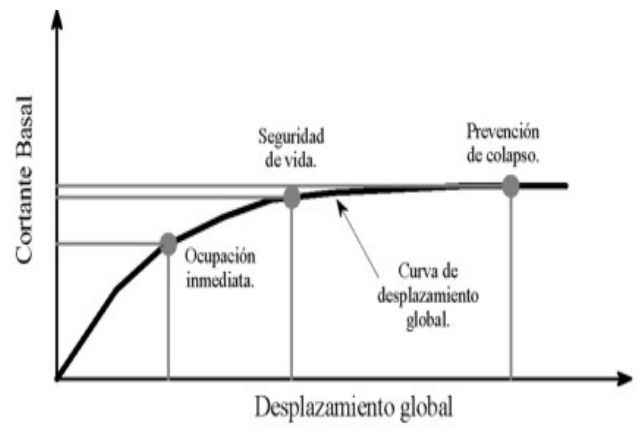

Figura 10. Punto de desempeño modelo 1

Finalmente se presentan (tabla 2) los puntos de desempeño obtenidos y el nivel de desempeño de cada uno de los modelos, teniendo en cuenta que los modelos colombianos tienen como límite de desempeño común "Seguridad de Vida" (LS), lo que determina que el uso de esta normativa da como resultado estructuras menos vulnerables, ante sismos, que sus pares ecuatoriana y peruana.

Tabla 2. Puntos y nivel de desempeño

\begin{tabular}{|c|c|c|c|c|}
\hline \multirow[t]{2}{*}{ PAIS } & \multirow{2}{*}{$\begin{array}{l}\text { MODELO } \\
\text { MODEL0 } 1\end{array}$} & \multicolumn{2}{|c|}{$\begin{array}{c}\text { PUNTO DE DESEMPEÑO } \\
(\mathrm{Sa} ; \mathrm{Sd})\end{array}$} & \multirow{2}{*}{$\begin{array}{l}\text { NIVEL DE DESEMPEÑO } \\
\text { Seguridad de Vida (LS) }\end{array}$} \\
\hline & & 0.234 & 0.087 & \\
\hline \multirow{8}{*}{$\varepsilon^{\circ}$} & MODELO 2 & 0.233 & 0.096 & Seguridad de Vida (LS) \\
\hline & MODELO 3 & 0.261 & 0.222 & Prevención de Colapso (CP) \\
\hline & MODELO 4 & 0.252 & 0.157 & Seguridad de Vida (LS) \\
\hline & MODELO 5 & - & - & Prevención de Colapso (CP) \\
\hline & MODELO 6 & 0.148 & 0.0564 & Seguridad de Vida (LS) \\
\hline & MODELO 7 & 0.159 & 0.057 & Seguridad de Vida (LS) \\
\hline & MODELO 8 & 0.175 & 0.181 & Prevención de Colapso (CP) \\
\hline & MODELO 9 & - & - & Prevención de Colapso (CP) \\
\hline \multirow{5}{*}{$0^{2}$} & MODELO 10 & 0.236 & 0.055 & Seguridad de Vida (LS) \\
\hline & MODELO 11 & 0.318 & 0.066 & Seguridad de Vida (LS) \\
\hline & MODELO 12 & 0.307 & 0.108 & Seguridad de Vida (LS) \\
\hline & MODELO 13 & 0.310 & 0.135 & Seguridad de Vida (LS) \\
\hline & MODELO 14 & 0.240 & 0.352 & Prevención de Colapso (CP) \\
\hline \multirow{4}{*}{$\beta^{\Delta \nu}$} & MODELO 15 & 0.224 & 0.032 & Seguridad de Vida (LS) \\
\hline & MODELO 16 & 0.265 & 0.061 & Seguridad de Vida (LS) \\
\hline & MODELO 17 & - & - & Prevención de Colapso (CP) \\
\hline & MODELO 18 & - & - & Prevención de Colapso (CP) \\
\hline
\end{tabular}

\section{CONCLUSIONES}

La aplicación de la normativa colombiana da como resultado estructuras con mejores niveles de desempeño dentro del marco de este análisis comparativo. Dado que los puntos de desempeño obtenidos con la NSR-10 (Colombia) son más conservadores respecto al resto de casos.

Se considera que, al tener valores de derivas de piso altas, los elementos no estructurales tendrán un daño importante, mientras que al rigidizar los elementos estructurales se tendrá derivas con valores más bajos y por lo tanto se reduciría el daño en los elementos no estructurales. La figura 11 muestra una comparativa de las secciones obtenidas, se aprecia que utilizando la normativa peruana las secciones son mayores para un mismo edificio, así mismo se evidencia que las secciones de columnas y vigas de acuerdo a la normativa colombiana son 1.85 y 1.59 respectivamente veces más que la norma ecuatoriana, y con la normativa peruana son 2.40 y 3.56 respectivamente veces más, lo que significa que la aplicación de esta última produce edificaciones más rígidas.

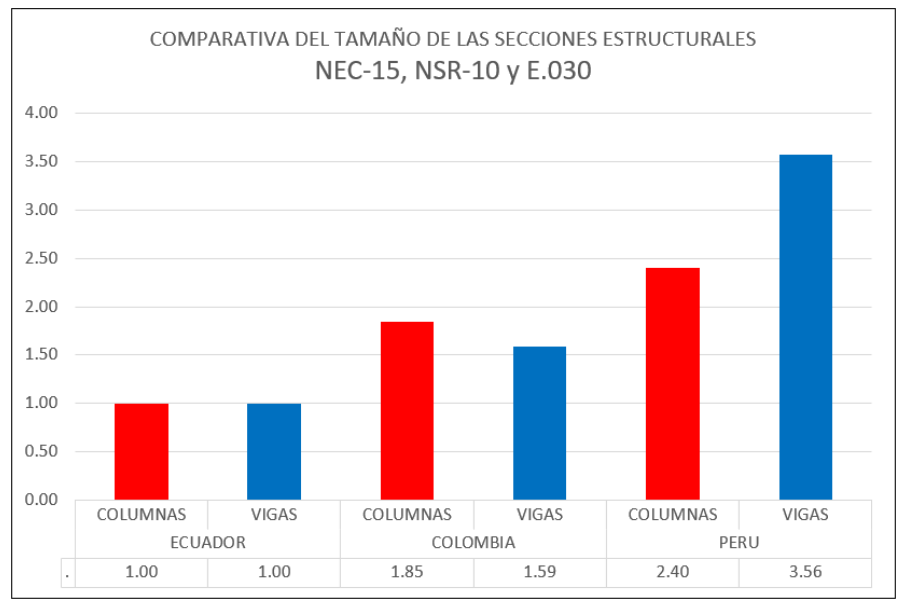

Figura 11. Comparativa de tamaño de secciones.

En el diseño de la estructura tanto la NEC15, la NSR-10 y la E-030 establecen que los elementos estructurales serán agrietados; en Ecuador, Colombia y Perú los factores de reducción de rigidez son diferentes, por este motivo los modelos obtenidos usando la norma colombiana tienen un mejor desempeño pese a ser menos rígidos que los modelos obtenidos usando la norma peruana, sin embargo se aprecia que tanto las norma colombiana como la peruana muestran mejores niveles de desempeño que los obtenidos con la norma ecuatoriana, se concluye que la deriva máxima establecida en la NEC-15 es demasiado permisiva e incide directamente en el nivel de desempeño obtenido. 


\section{REFERENCIAS}

Aguiar, R. (2003). Análisis sísmico por desempeño, Centro de Investigaciones Científicas, Escuela Politécnica del Ejercito, Quito, Ecuador.

Agostini, M. y Gerbaudo, G.M. (2018). Análisis Estático No-Lineal para Cuantificar la Vulnerabilidad Sísmica de Edificios de Hormigón Armado, Universidad Nacional de Córdoba. Córdoba, Argentina.

Andino, J.L. y Lema, C. (2020). Análisis Comparativo del Diseño Estructural de una Edificación de Cinco Pisos, de Hormigón Armado, Aplicando la Norma Ecuatoriana de la Construcción (NEC-2015), El Reglamento Colombiano de la Construcción (NSR-10) y El Reglamento Nacional de Edificaciones (E.030) Perú. (Tesis de Pregrado). Universidad Central del Ecuador. Quito, Ecuador.

Asociación Colombiana de Ingeniería Sísmica (S.F.). Reglamento Colombiano de Construcción Sismorresistente (NSR-10). Bogotá, Colombia.

Correa, M. Machado, L. (2016). Comparación Económica al Diseñar Edificios de Hormigón Armado de 7 y 14 Pisos, con la NEC-11 y la CEC2000. (Tesis de Postgrado). Escuela Politécnica Nacional. Quito, Ecuador.

Crisafulli, F. J. (2013). Diseño Sismorresistente de Construcciones de Acero. Mendoza, Argentina.

Duarte, C., Martínez, M., Santamaria J., (2017). Análisis Estático No Lineal (Pushover) del Cuerpo Central del Edificio de la Facultad de Medicina de la Universidad de El Salvador. El Salvador.

Durán, J. (2017). Comparación de las normas ASCE 7-10 y NEC-15 en el Diseño Sísmico con la aplicación a pórticos especiales con y sin Muros Estructurales. (Tesis de pregrado). Universidad de Cuenca.

Guía práctica para el diseño de estructuras de hormigón armado, de conformidad con la norma ecuatoriana de la construcción NEC 2015.

Guzmán M. F. (2015). Diseño Sismo Resistente de Edificios de Hormigón Armado, Cálculo de Periodos de Vibración y Niveles de Agrietamiento. (Tesis de pregrado). Pontificia Universidad Católica del Ecuador. Quito, Ecuador.

https://www.google.com.ec/search?sxsr$\mathrm{f}=\mathrm{ALeKk00ZdFOITGS5n5f112XeQ4Q6mr-}$ VHEA:1582829309635\&q=mapa+de+zonificaci\%C3\%B3n+s\%C3\%ADsmica+del+ecuador\&tbm=isch\&source=univ\&sa $=X \& v e d=2 a h U K E w j A m f b V s v L n A-$ hVJMawKHdh2Cn4QsAR6BAgKEAE\&biw=1536\&bi$\mathrm{h}=682 \& \mathrm{dpr}=1.25 \#$ imgrc=T6ZSpxipQ-tHQM

https://www.google.com.ec/search?q=mapa+de+zonificaci\%C3\%B3n+s\%C3\%ADsmica+del+peru\&tb$m=i s c h \& v e d=2 a h U K E w j-x O 3 Y$ svLnAhVPI1kKHSX5DoMQ2-cCegQIABAA\&oq=mapa+de+zonificaci\%C3\%B3n+s\%C3\%ADsmica+del+peru\&gs_l= img.3...39052.41351..42107 ...0.0..0.348.2909.0j1j6j4..........1..gws-wiz-img......35i39jo.M46n2SA2e3U\&ei=Ag9YXv69DM_G5AKI8ruYCA\&bih=682\&bi$w=1536 \#$ imgrc $=g E m W W v-X X C C O p M$

https://uww.google.com.ec/search?q=mapa+de+zonificaci\%C3\%B3n+s\%C3\%ADsmica+del+colombia\&tb$m=i s c h \& v e d=2 a h U K E w i A u P H s s v L n A h U K k 1 k K H Y 4 L-$ CrwQ2-cCegQIABAA\&oq=mapa+de+zonificaci\%C3\%B3n+s\%C3\%ADsmica+del+colombia\&gs_l= img.3..35i39.13215.15361..15759...0.0..0.389.3502.0j1j4j7..........1..gws-wiz-img.......0i67j0.HyUP9FPOOlo\&ei=LQ9YXsDtL4qm5gKOl6jgCw\&bih=682\&bi$\mathrm{w}=$ 1536\#imgrc=RzAc5Ecbq_22MM.

Ministerio de Desarrollo Urbano y Vivienda, Norma Ecuatoriana de la Construcción (NEC-2015), NEC-SE-DS. Peligro Sísmico, Diseño Sismo Resistente, Quito, Ecuador.

Reglamento Colombiano de Construcción (NSR-10). Titulo A, Requisitos generales de diseño y construcción sismo resistente. Bogotá, Colombia.

Reglamento Nacional de Edificaciones (E-030) PERU.

Vasco, P. (2016). Sismo Resistencia en Edificios de Hormigón y Acero para Zonas de Alta Peligrosidad Sísmica. (Tesis de pregrado). Universidad Técnica de Ambato. Ambato, Ecuador. 